\title{
Aminobenzoate Potassium
}

National Cancer Institute

\section{Source}

National Cancer Institute. Aminobenzoate Potassium. NCI Thesaurus. Code C1736.

The potassium salt form of aminobenzoate, with anti-inflammatory and antifibrotic activities. Aminobenzoate potassium increases oxygen uptake at the tissue level and may enhance monoamine oxidase (MAO) activity, which requires oxygen as a substrate.

Enhanced MAO activity maybe accountable for the prevention or regression of fibrosis, which may occur due to too much serotonin or too little MAO activity. 